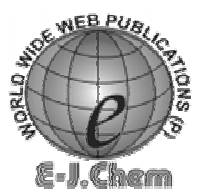

http://www.e-journals.net
ISSN: 0973-4945; CODEN ECJHAO

E-Journal of Chemistry

Vol. 5, No. 2, pp.316-322, April 2008

\title{
Thermal and Isothermal Methods in Development of Sustained Release Dosage Forms of Ketorolac Tromethamine
}

\author{
DIMPLE CHOPRA*, VIVEK RANJAN SINHA and MANJEET SINGH \\ Department of Pharmaceutical Sciences and Drug Research \\ Punjabi University, Patiala -147002 \\ dimplechopra24@yahoo.co.in
}

Received 30 September 2007; Accepted 20 November 2007

\begin{abstract}
Differential scanning calorimetry (DSC) is a rapid and convenient and conclusive method of screening drug-polymer blend during preformulation studies as it allows polymer incompatibility to be established instantaneously. Various batches of matrix tablets of ketorolac tromethamine (KTM) with a series of compatible polymers were prepared. Batches of tablets which gave desired sustained release profile were subjected to stability testing according to ICH guidelines. The analysis for drug content was done using high performance liquid chromatography (HPLC) method. The results revealed that there was no statistically significant change in drug content after storage of matrix tablets at elevated temperature of $40{ }^{\circ} \mathrm{C}$ and $75 \%$ relative humidity. From our study we conclude that with careful selection of different polymers and their combinations, a stable sustained release oral dosage form of ketorolac tromethamine can be achieved.
\end{abstract}

Keywords: Ketorolac tromethamine, DSC, HPLC, matrix tablets, stability testing.

\section{Introduction}

Ketorolac tromethamine (KTM) is a non steriodal anti-inflammatory drug which exhibits potent analgesic, anti-inflammatory and antipyretic ${ }^{1-3}$ effects. It is administered intramuscularly, intravenously, orally and as ocular ${ }^{4}$ formulation. KTM is a basic salt of a moderately acidic drug (pKa 3.49), with a reasonable solid-state stability. The excipientmediated decomposition of ketorolac in the solid state depends on salt form of the drug, the type of excipient, moisture content, temperature and oxygen pressure. In solid blends, ketorolac undergoes decarboxylation, oxidation and amidation to form decarboxy analog, 1-keto analog or 1-hydroxy analog and amide of ketorolac ${ }^{5}$ respectively. Several approaches have been identified in literature for improving the stability ${ }^{6,7}$ of ketorolac powder blends. To predict shelf-life of the final dosage form one should know about stability of the active 
ingredient and other components of the formulation. Most of the techniques ${ }^{8}$ reported in the literature are labor intensive, time consuming, deal with limited number of variables and have poor predictive values. The studies on the interaction between the drug and the excipients are generally carried out by means of DSC or accelerated stability tests followed by analytical determination ${ }^{9}$ (HPLC and other methods) of the active principle. There are conflicting reports in literature about compatibility of certain excipients and ketorolac tromethamine upon individual application of these two analytical methods. Therefore the present paper stresses upon the need of using both thermal and isothermal methods in development of stable and efficacious drug formulations.

\section{Experimental}

Ketorolac tromethamine (Ranbaxy Laboratories Ltd, Gurgaon), Methocel (Colorcon Asia Pvt. Ltd, Mumbai), Xanthan gum USP (Dabur Research Foundation, Sahibabad), Carbopol974P (Noveon, Mumbai) and Eudragit (S. Zhaveri \& Co., Mumbai) were obtained as a gift sample. The solvents used for HPLC analysis were purchased from different commercial sources. All the other excipient used were of AR grade. De-ionized water was used throughout the study.

\section{Instruments}

WTC binder stability chamber (Germany) was used to carry out stability studies. DSC (DSC $821^{\mathrm{e}}$, Metler Toledo) was used for thermal analysis. HPLC was performed on Shimadzu systems fitted with a LC-10 ATVP pumping system, SPD-10AVP UV-Vis detector (at 254 $\mathrm{nm})$ with class VP software and ODS reverse-phase $\mathrm{C}_{18}(25 \mathrm{~cm} \mathrm{X} 4.5 \mathrm{~mm})$ column.

\section{Drug-polymer compatibility studies}

Powder blends (50mg) of drug and various polymers i.e HPMC, Carbopol 974P, xanthan gum were prepared using geometric dilution method. The ratio of drug to polymer chosen was same as that in the final formulation. DSC was calibrated by using indium as a standard with melting point $\mathrm{T}_{\text {fus }}$ at $156.63{ }^{\circ} \mathrm{C}$ and calibration energy $\left[\Delta \mathrm{H}_{\text {fus }}\right]$ of $28.89 \mathrm{~J} / \mathrm{g}$. Accurately weighed samples (5mg) were heated in sealed aluminium pans from 25 to $300{ }^{\circ} \mathrm{C}$ at a scanning rate of $10{ }^{\circ} \mathrm{C} / \mathrm{min}$ under nitrogen purging $(80 \mathrm{~mL} / \mathrm{min})$. Differential scanning calorimetry was conducted first with samples of the pure polymers, pure drug and then on prepared drug-polymer blends. The DSC profiles thus obtained were compared for possible drug-polymer interactions.

\section{Stability studies}

ICH stability guidelines ${ }^{10}$ were strictly followed to carry out stability studies. All the matrix tablets were prepared by direct compression. The general formula for matrix tablets is shown in Table 1. Four batches of hydrophilic matrix tablets containing $80 \% \mathrm{w} / \mathrm{w}$ of polymers ie HPMCK4M, Carbopol974P, Carbopol974P:HPMC combinations (1:1 and 1:3) were kept in a screw capped amber coloured glass bottles. All the glass bottles were packed in a black canvas bag and placed in the stability chamber at $40{ }^{\circ} \mathrm{C}$ and $75 \% \mathrm{RH}$ for six months. Samples were withdrawn at periodic time intervals i.e 1,2,3 and 6 months and tested for drug content using HPLC method.

\section{Preparation of calibration curve for HPLC analysis}

An accurately weighed quantity (100mg) of KTM was transferred to a clean, dry $100 \mathrm{~mL}$ volumetric flask. The sample was dissolved in water $(50 \mathrm{~mL})$ and the final volume was adjusted to $100 \mathrm{~mL}$ and labelled as stock solution. The stock solution was diluted with mobile phase (water:methanol:acetic acid 40:59:1) to produce solutions with concentration range of $1.0-20.0 \mathrm{mcg} / \mathrm{mL}$. Twenty microlitres of each dilution was injected triplicate into the 
chromatographic system. The peak area was used as the response for the preparation of the calibration curve. The method was validated for linearity and accuracy.

\section{Quantitative analysis of the stressed samples}

All isothermally stressed samples were analyzed by reported ${ }^{6}$ HPLC method. To an accurately weighed sample of crushed tablets, $15 \mathrm{~mL}$ of water was added and the mixture was processed ultrasonically for further 10min. This procedure was followed in order to solubilize all the drug and its degradation products (if formed), leaving the insoluble excipients. A $5 \mathrm{~mL}$ portion of this solution was filtered through a $0.45 \mu \mathrm{m}$ membrane filter. An aliquot of filtrate $(1 \mathrm{~mL})$ was diluted with the mobile phase (dilution factor 25) and analyzed by HPLC. The percentage of residual KTM was determined using peak area of KTM from the calibraton curve.

Table 1. Formulation of ketorolac tromethamine used in the study.

\begin{tabular}{lc}
\hline \multicolumn{1}{c}{ Ingredient } & Milligrams per tablet \\
\hline Ketorolac tromethamine & 10 \\
Polymer & 160 \\
Spray-dried lactoseUSP & 26 \\
Talc & 2 \\
Magnesium stearate & 2 \\
\hline
\end{tabular}

\section{Results and Discussion}

The DSC thermograms of the drug and polymers tested are shown in Figures 1-5. Each of these figures indicates the thermal behavior pattern of the pure drug, polymer and drug-polymer blend. The DSC trace of KTM shows three endothermic peaks at $160.94,167.85,196.73{ }^{\circ} \mathrm{C}$. The first peak is associated with the melting of KTM (reported m.p is $160-161{ }^{\circ} \mathrm{C}$ ). The second and third endothermic peaks are related to the decomposition and evaporation of the drug respectively. In all drug-polymer blend, the melting endotherm of KTM was well preserved with little change in terms of sharpening, broadening or shifting towards a lower temperature. These minor changes in the melting endotherm of the drug may be attributed to mixing process, which lowers the purity of each component in the mixture, thus resulting in slightly lower melting points, but not truly representative of incompatibility. Slight variations in the peak shape and melting point may be also triggered by varying sample geometry ${ }^{11}$ during mixing. In some cases, the shape of the DSC curve of the pure excipient differed from that of the mixture. This may be due to variations in the quantities of excipient used for the analysis. The peak shape and enthalpy depend on quantity of material used whilst the peak transition temperature ${ }^{12,13}$ associated with complete fusion is independent. Since the amount of polymer present in the corresponding mixture is less than that in the pure substance. The difference in peak shape was apparent. Therefore, peak transition temperatures were taken into account for interpretation of DSC curves. The polymers did not show any characteristic endotherms, instead a broad endotherm was observed below $100{ }^{\circ} \mathrm{C}$. This is attributed to evaporation of absorbed water from the polymers during heating.

The melting endotherm of the drug was well preserved in all cases apart from a considerable shift to a lower temperature in the case of HPMC. The peak corresponding to decomposition of KTM was missing in the blend of KTM with Carbopol 974P and HPMC K4M (1:1) as compared to KTM with Carbopol 974P and HPMC K4M (1:3). This can be very well explained by considering the DSC thermogram of blend of KTM and Carbopol 974P, both the melting and decomposition endotherms are very much suppressed. So as the proportion of Carbopol increases in a drug polymer blend its protective effect on the drug molecule increases. So, KTM is chemically compatible with all these polymers. 


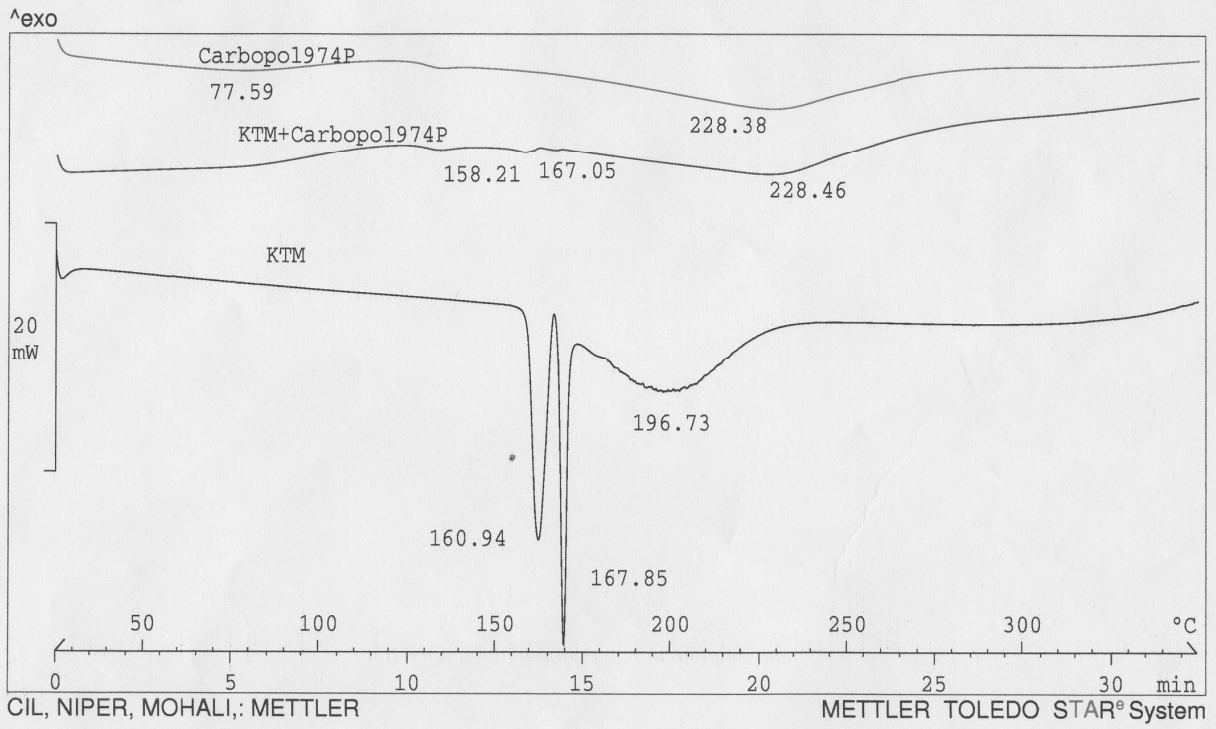

Figure 1. DSC thermograms of KTM with Carbopol 974P

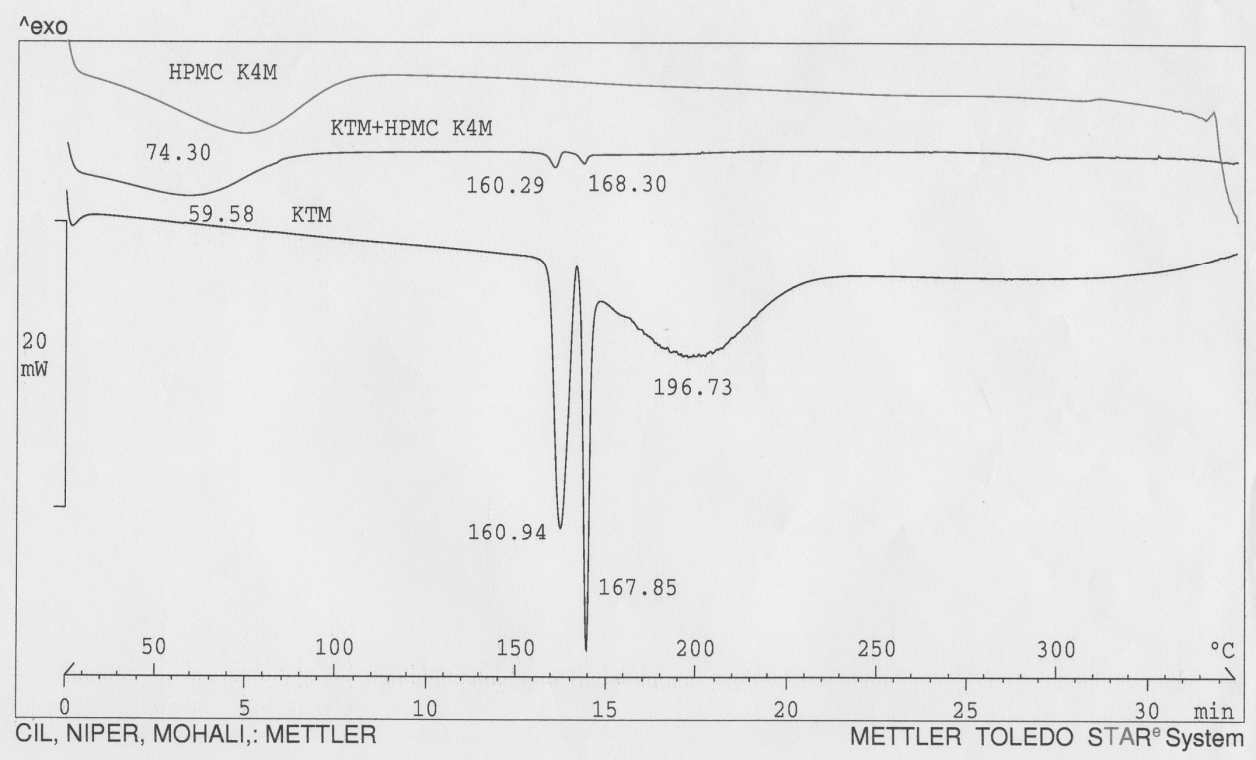

Figure 2. DSC thermograms of KTM with HPMC K4M 


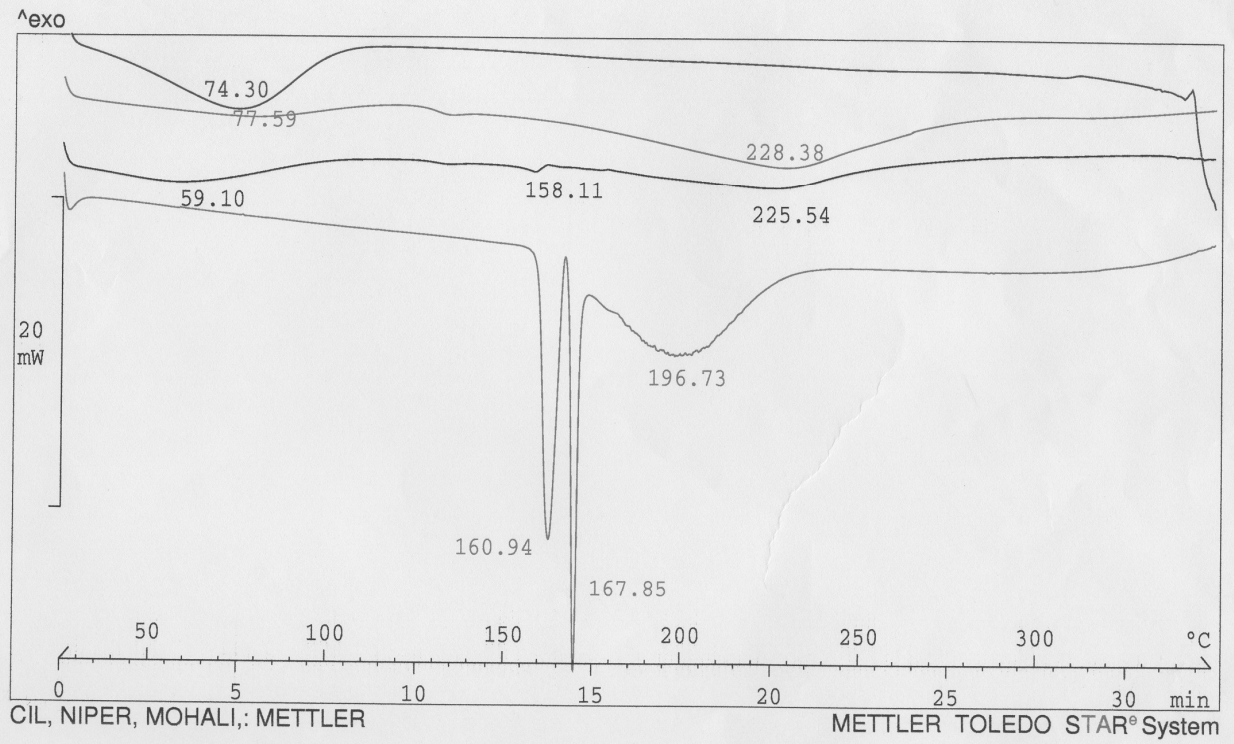

Figure 3. DSC thermograms of KTM with HPMC K4M and Carbopol 974P (1:1)

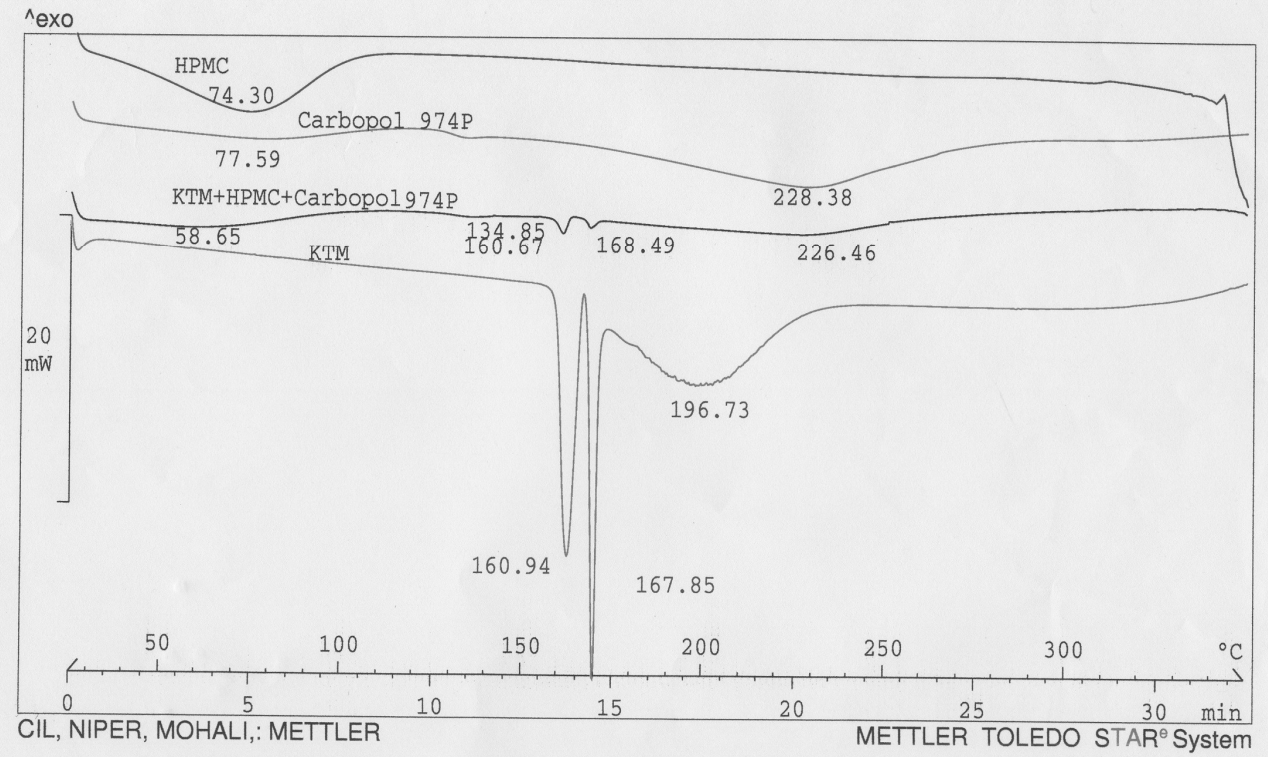

Figure 4. DSC thermograms of KTM with HPMC K4M and Carbopol 974P (1:3) 


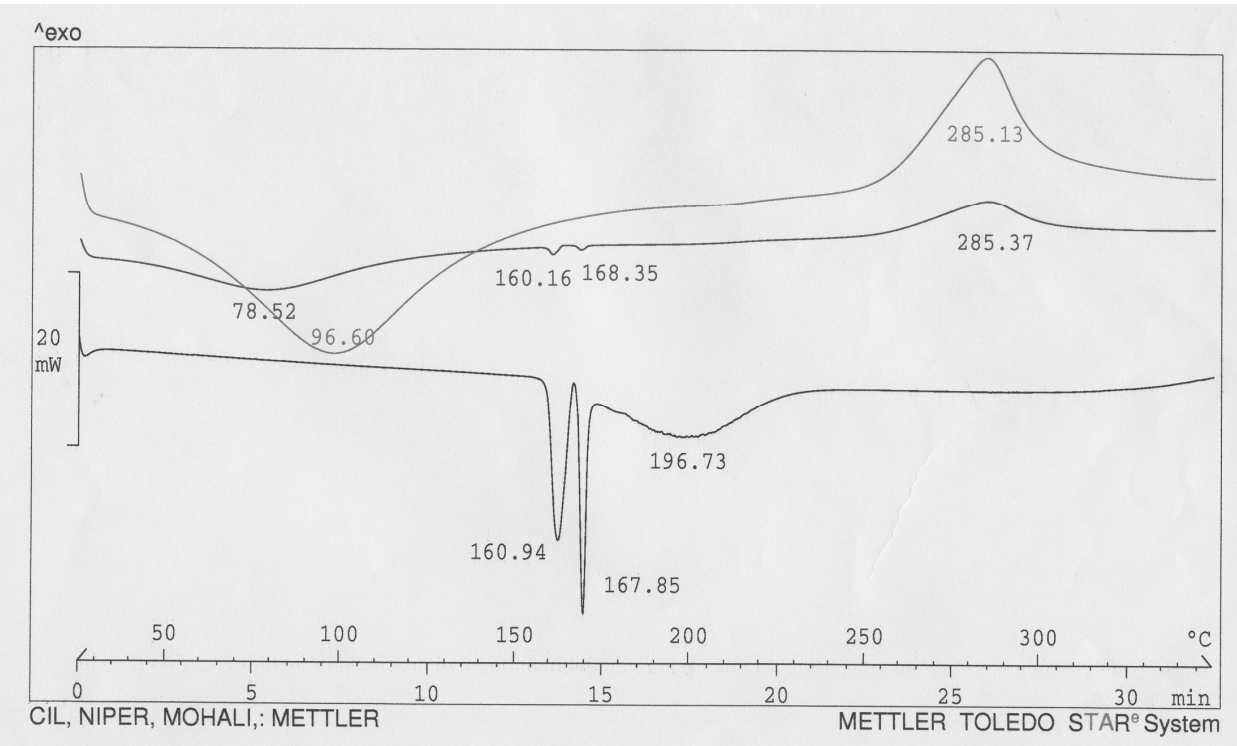

Figure 5. DSC thermograms of KTM with xanthan gum

From the typical chromatogram of KTM as shown in Figure 6, it was found that the retention time was $8.296 \mathrm{~min}$. A good linear relationship $(\mathrm{r}=0.9850)$ was observed between the concentration range of $1.0-20.0 \mathrm{mcg} / \mathrm{mL}$. The absence of additional peaks in the chromatogram indicates non-interference of the common excipients used in the tablets. This demonstrates that the HPLC method used is simple, linear, accurate, sensitive and reproducible. The summary of analytical results of stressed samples is given in Table 2. In all the stressed samples, the percentage drug recovered was more than $94 \%$ after a period of one month. These polymers are Class I type excipients, which are known to exhibit negligible chemical compatibility and stability problems. Even after exposure to stressed conditions for a period of 6 months the drug content in all the samples was above 90\%. This shows that KTM is chemically compatible with various excipients used in the formulation. The results of DSC and HPLC were in consonance for all the drug-polymer blends used in the study. This confirmed the utility of DSC as a rapid and convenient method for screening various excipients during preformulation stage. Further, quantitative assessment ${ }^{9}$ can be made using isothermal methods like HPLC. DSC can also be combined with spectral techniques (diffused reflectance spectroscopy, FTIR) to elucidate ${ }^{12,13}$ degradation mechanisms.

Table 2. Summary of HPLC assay of isothermally stressed samples of KTM with various polymers

\begin{tabular}{lrccc}
\hline \multicolumn{1}{c}{ Polymer(s) } & \multicolumn{4}{c}{ Percentage drug recovered } \\
\cline { 2 - 5 } \multicolumn{1}{c}{ used } & 1 month & 2 months & 3 months & 6 months \\
\hline Carbopol 974P & 98.4 & 96.7 & 95.0 & 94.0 \\
HPMC K4M & 97.3 & 94.7 & 94.3 & 93.2 \\
Carbopol:HPMC(1:1) & 97.6 & 95.0 & 94.6 & 93.8 \\
Carbopol:HPMC(1:3) & 96.5 & 95.0 & 94.6 & 90.7 \\
Xanthan gum & 99.9 & 97.8 & 95.1 & 95 \\
\hline
\end{tabular}




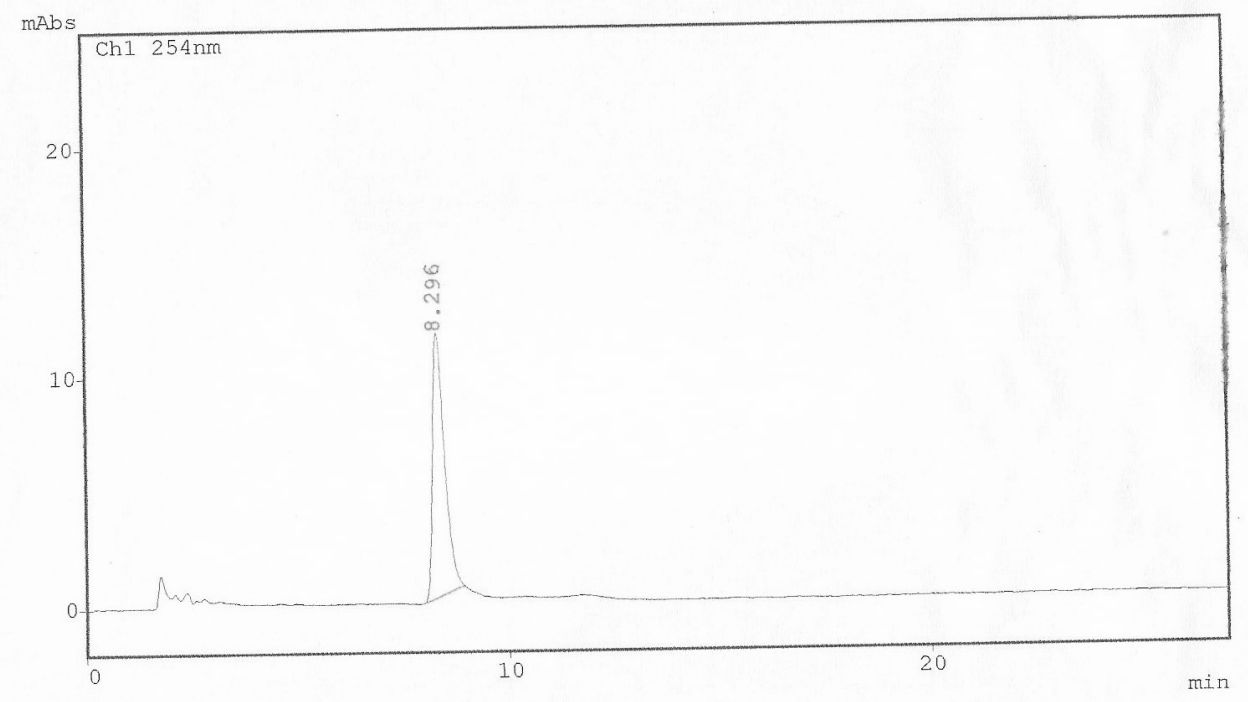

Figure 6. Typical Chromatogram of KTM by HPLC

\section{References}

1. Buckley MM and Brogden RN, Drugs, 1990, 39, 86-109.

2. $\quad$ Brocks DR and Jamali F, Clin. Pharmacokinet., 1992, 23, 415-427.

3. Cho YA and Gwak HS, Drug Dev. Ind. Pharm. 2004, 30, 87-194.

4. $\quad$ Sinha VR and Trehan A, Drug Deliv., 2005, 12, 133-139.

5. Gu L, Chiang HS and Becker A, Int. J. Pharm., 1998, 41, 95-104.

6. Brandl M, Magill A, Rudraraju V and Gordon M S, J. Pharm. Sci., 1995, 84,1151.

7. Brandl M, Conley D and Johnson D, J. Pharm. Sci., 1995, 84, 1045- 1048.

8. Marini A, Berbenni V, Bruni G, Cofrancesco P, Giordano F and Villa M, Curr. Med. Chem. 2003, 2, 303-321.

9. Ceschel GC, Badiello R, Ronchi C and Maffei P, J. Pharm. Biomed. Anal., 2003, 32,1067-1072.

10. Singh S, Pharm. Technol. 1999, 23, 68-88.

11. Kandarapu R, Grover V, Chawla HPS and Garg S, S.T.P. Pharm. Sci., 2001, 11, 449.

12. Mura P, Faucci M T, Manderioli A, Furlanetto S and Pinzauti S, Drug Dev. Ind. Pharm., 1998, 24, 747-756.

13. Swarbrick J and Boylan J C, Encyclopedia of Pharmaceutical Technology, Marcel Dekker, New York, 1996,1-79. 


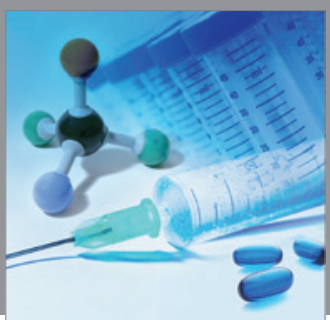

International Journal of

Medicinal Chemistry

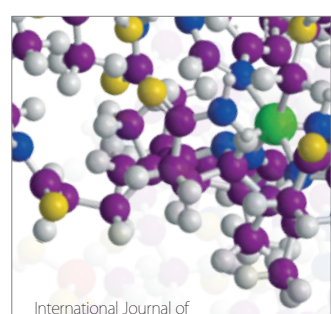

Carbohydrate Chemistry

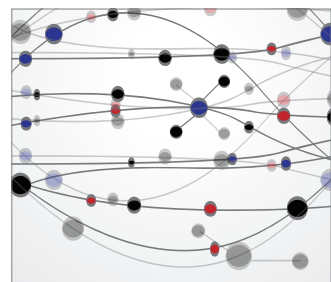

The Scientific World Journal
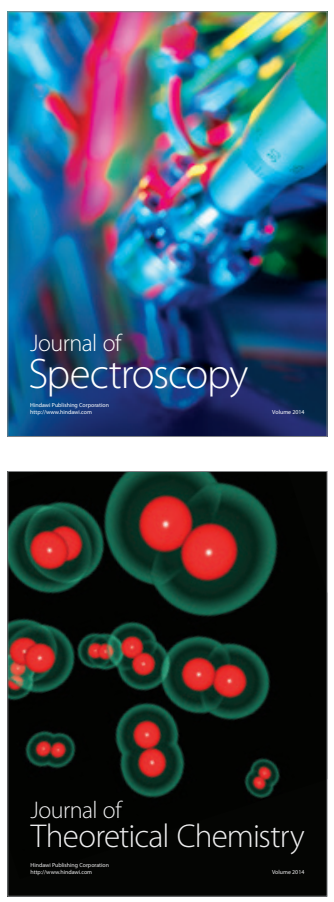
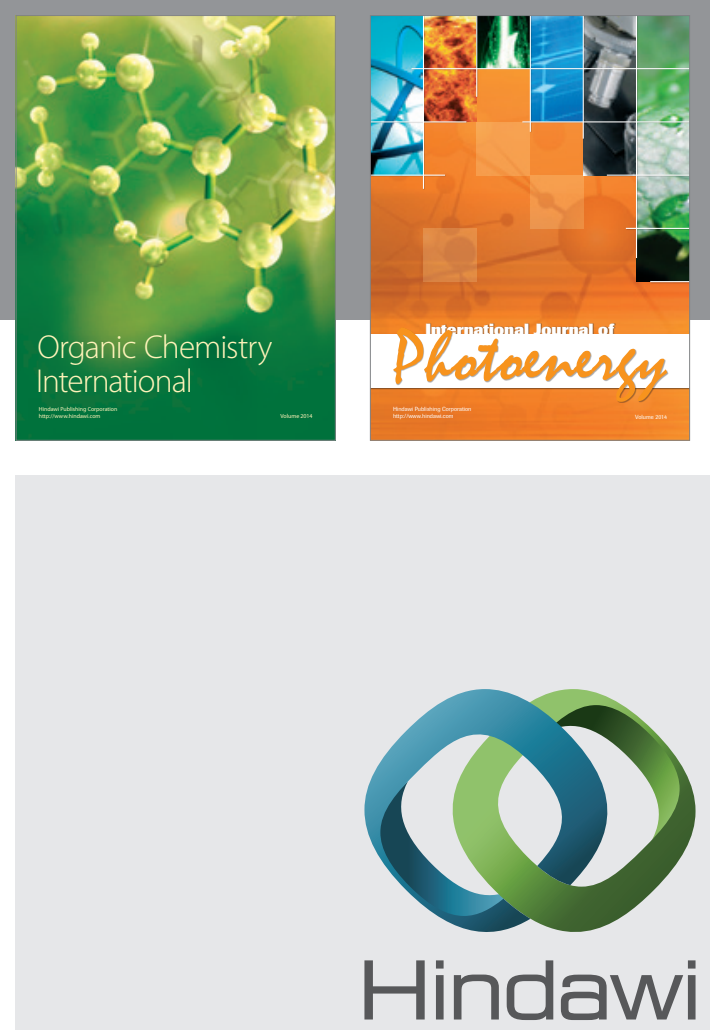

Submit your manuscripts at

http://www.hindawi.com
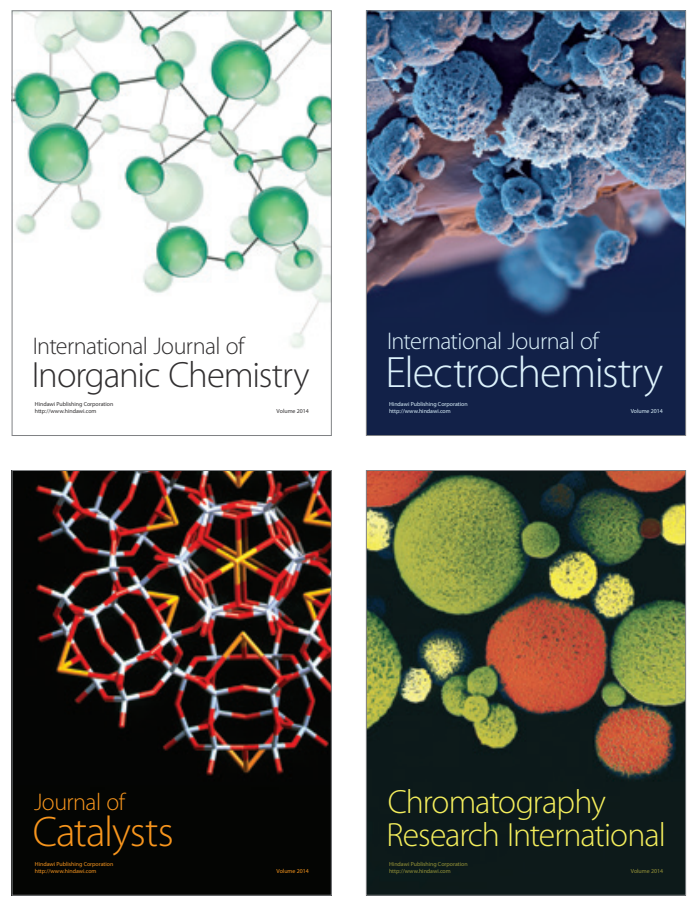
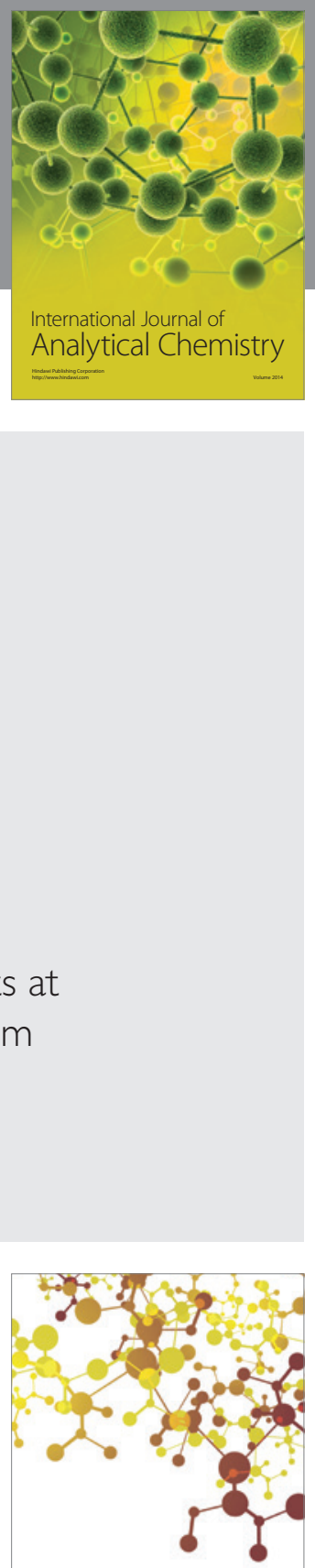

Journal of

Applied Chemistry
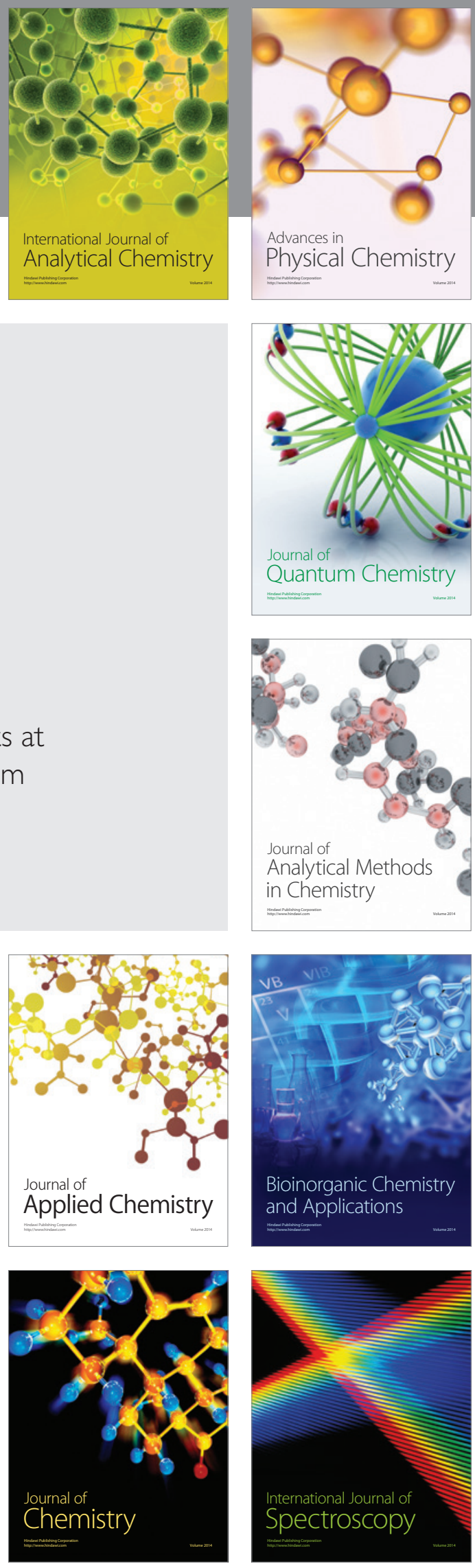